\title{
An adaptive central-upwind scheme on quadtree grids for variable density shallow water equations
}

\author{
Mohammad A. Ghazizadeh*, Abdolmajid Mohammadian \\ Department of Civil Engineering, University of Ottawa, Ottawa, ON K1N 6N5, Canada
}

\begin{abstract}
Minimizing computational cost is one of the major challenges in the modelling and numerical analysis of hydrodynamics, and one of the ways to achieve this is by the use of quadtree grids. In this paper, we present an adaptive scheme on quadtree grids for variable density shallow water equations. A scheme for the coupled system is developed based on the well-balanced positivity-preserving central-upwind scheme proposed in [18]. The scheme is capable of exactly preserving "lake-at-rest" steady states. A continuous piecewise bi-linear interpolation of the bottom topography function is used to achieve higher-order in space in order to preserve the positivity of water depth for the point values of each computational cell. Necessary conditions are checked to be able to preserve the positivity of water depth and density, and to ensure the achievement of a stable numerical scheme. At each timestep, local gradients are examined to find new seeding points to locally refine/coarsen the computational grid.
\end{abstract}

Keywords: Shallow water equations, Variable density, Quadtree grids, Central-upwind scheme, Well-balanced scheme, Positivity-preserving scheme.

\section{Introduction}

Quadtree grids are two-dimensional (2-D) semi-structured Cartesian grids that can be very accommodating for various problems in the field of computational hydrodynamics. One of the advantages of quadtree grids over structured and unstructured grids is grid coarsening/refining. The accuracy is increased/maintained while the grid refines/coarsens wherever it is needed and thus, the computational cost is reduced. There are a number of studies on how to generate quadtree grids; see, e.g., [1, 9, 18, 21, 39, 41, 44, 45].

The main goal of this paper is to develop an adaptive well-balanced positivity-preserving central-upwind scheme on quadtree grids for the coupled variable density shallow water equations (SWEs). The variable density SWEs in 2-D can be written in terms of conservative variables of

\footnotetext{
*Corresponding author.

Email addresses: sghaz023@uottawa.ca (Mohammad A. Ghazizadeh), majid.mohammadian@uottawa.ca (Abdolmajid Mohammadian)
} 
$w$ (water surface), $h u$ and $h v$ (the unit discharges), and $h \rho$ :

$$
\left\{\begin{array}{l}
w_{t}+(h u)_{x}+(h v)_{y}=0, \\
(h u)_{t}+\left(h u^{2}+\frac{g}{2 \rho_{\circ}} h^{2} \rho\right)_{x}+(h u v)_{y}=-\frac{g}{\rho_{\circ}} h \rho B_{x}, \\
(h v)_{t}+(h u v)_{x}+\left(h v^{2}+\frac{g}{2 \rho_{\circ}} h^{2} \rho\right)_{y}=-\frac{g}{\rho_{\circ}} h \rho B_{y}, \\
(h \rho)_{t}+(h u \rho)_{x}+(h v \rho)_{y}=0,
\end{array}\right.
$$

where $t$ is time, $g$ is the gravitational constant, $x$ and $y$ are the directions in the 2-D Cartesian coordinate system, $u(x, y, t)$ and $v(x, y, t)$ are the water velocities in the $x$ - and $y$-directions, respectively, $B(x, y)$ is the bottom topography, $h(x, y, t)=w(x, y, t)-B(x, y)$ is the water depth, $\rho$ is the density, and $\rho_{\circ}$ is the reference density.

System (1.1) admits "lake-at-rest" steady-state solutions,

$$
\rho \equiv \text { Const }, \quad w \equiv \text { Const }, \quad u=v \equiv 0, \quad B \equiv \text { Const },
$$

which can be obtained from (1.1) [15]. The following quadtree scheme is capable of exactly preserving "lake-at-rest" steady states, which is called the well-balanced property. Another important attribute of the following method is its ability to preserve the non-negativity of $h$ and $\rho$, which is called the positivity-preserving property (see [26] for a comprehensive review on these subjects).

A number of numerical schemes on quadtree grids for the SWEs have been introduced in recent years. For example, an adaptive well-balanced positivity-preserving central-upwind highorder scheme on quadtree grids was proposed in [18]. In addition, a well-balanced scheme on quadtree-cut-cell grids was proposed in [2]. This scheme is based on the hydrostatic reconstruction from [4]. Furthermore, an adaptive second-order Roe-type scheme was proposed in [43]. An adaptive well-balanced Godunov-type scheme for the shallow water for the wet-dry over complex topography was introduced in [36] and an adaptive quadtree Roe-type scheme for 2-D two-layer SWEs was presented in [33]. Besides the aforementioned numerical methods, several well-balanced positivity-preserving central-upwind schemes for the shallow water equations have been proposed in the past years; see, e.g., [3, 4, 5, 6, 7, 8, 10, 14, 13, 17, 27, 30, 37, 42, 46], yet, to our knowledge, none of these methods has been extended to the coupled variable density SWEs over quadtree grids.

In [24] the coupled variable density SWEs were studied with a Godunov-type HLLC approximate Riemann solver. There are other studies that have been conducted on variable density SWEs and variable horizontal temperature SWEs (which have mathematically similar properties) with different numerical schemes; see, e.g. [15, 22, 25, 34].

In this paper, we propose a central-upwind quadtree scheme which is based on the one from [18]. Central-upwind schemes are finite-volume methods that are Godunov-type Riemannproblem-solver-free [28, 29, 31, 32. Central-upwind schemes have been referred to as "black-box" solvers for general multidimensional systems of hyperbolic systems of conservation laws, and hav been extended to shallow water models [26]. The proposed scheme is the first well-balanced positivity-preserving central-upwind scheme for the variable density SWEs over quadtree grids. This method is simple, efficient, and robust. 
The paper is organized as follows. In \$2, we briefly describe the quadtree grid generation terminology. In $\S 3$, we construct a central-upwind quadtree scheme for the variable density SWEs with the mentioned features and test it on four different numerical examples in $\$ 4$. Finally in $\$ 5$, some concluding remarks are presented.

\section{Quadtree grids}

In this section, we denote the terminology for how to generate quadtree grids (see [18, 9, 21, 40]):

Seeding points: A set of points that helps to locally refine/coarsen the computational grid when needed.

Level of refinement: Level of the quadtree, in which the size of the smallest cell is inversely proportional to the maximum level of $m$.

Regularised quadtree grid: In a regularised grid, no cell can have both an adjacent neighboring cell and a diagonally neighboring cell with a refinement level difference greater than one (Figure 1). The proposed scheme is based on regularised quadtree grids to prevent complicated formulations and improve stability.
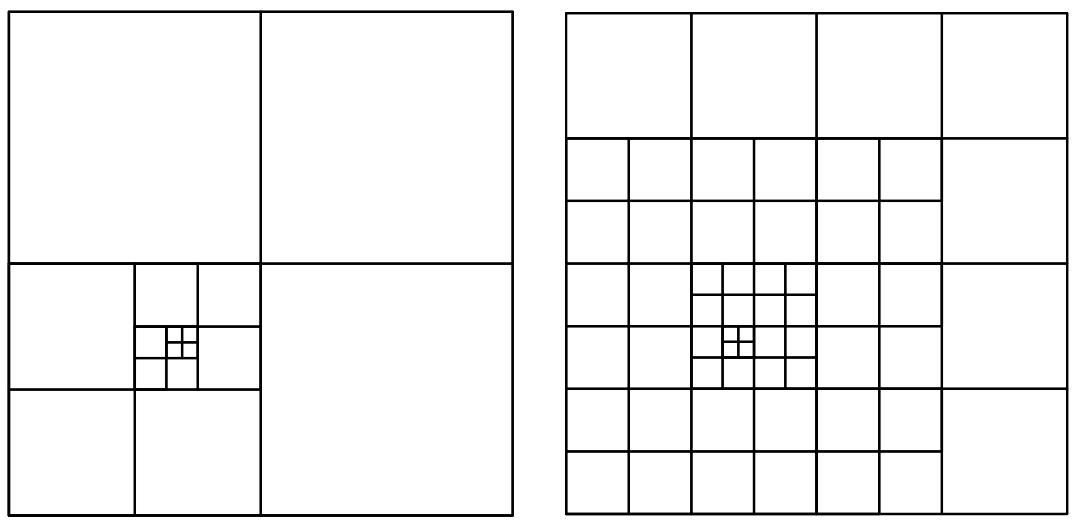

Figure 1: Examples of non-regularised (left) and regularised (right) quadtree grids.

\section{Adaptive semi-discrete central-upwind scheme}

We write system (1.1) in the following vector form:

$$
\boldsymbol{U}_{t}+\boldsymbol{F}(\boldsymbol{U}, B)_{x}+\boldsymbol{G}(\boldsymbol{U}, B)_{y}=\boldsymbol{S}(\boldsymbol{U}, B),
$$

where

$$
\boldsymbol{U}:=(w, h u, h v, h \rho)^{\top}
$$


and the fluxes and source term are:

$$
\begin{aligned}
& \boldsymbol{F}(\boldsymbol{U}, B)=\left(h u, \frac{(h u)^{2}}{w-B}+\frac{g}{2 \rho_{\circ}} \rho(w-B)^{2}, \frac{(h u)(h v)}{w-B}, h u \rho\right)^{\top}, \\
& \boldsymbol{G}(\boldsymbol{U}, B)=\left(h v, \frac{(h u)(h v)}{w-B}, \frac{(h v)^{2}}{w-B}+\frac{g}{2 \rho_{\circ}} \rho(w-B)^{2}, h v \rho\right)^{\top}, \\
& \boldsymbol{S}(\boldsymbol{U}, B)=\left(0,-\frac{g}{\rho_{\circ}} \rho(w-B) B_{x},-\frac{g}{\rho_{\circ}} \rho(w-B) B_{y}, 0\right)^{\top} .
\end{aligned}
$$

In the following, an adaptive well-balanced semi-discrete central-upwind scheme for $(3.1)$ is presented. The proposed scheme will be designed according to the algorithm in [18]:

\subsection{Finite-volume semi-discretization over quadtree grids}

Let us represent each cell $C_{j, k}$ of size $\Delta x_{j, k} \times \Delta y_{j, k}$ centered at $\left(x_{j, k}, y_{j, k}\right)$ as a finite volume quadtree cell in the proposed scheme. The approximate averages of the cell read as:

$$
\overline{\boldsymbol{U}}_{j, k}(t) \approx \frac{1}{\Delta x_{j, k} \Delta y_{j, k}} \int_{x_{j-\frac{1}{2}}}^{x_{j+\frac{1}{2}}} \int_{y_{k-\frac{1}{2}}}^{y_{k+\frac{1}{2}}} \boldsymbol{U}(x, y, t) \mathrm{d} y \mathrm{~d} x
$$

where $x_{j \pm \frac{1}{2}}:=x_{j, k} \pm \Delta x_{j, k} / 2$ and $y_{k \pm \frac{1}{2}}:=y_{j, k} \pm \Delta y_{j, k} / 2$. We present the proposed scheme for the configuration in Figure 2. The left-neighboring cells of $C_{j, k}$ are denoted by $I$ and $I I$ which are centered at $\left(x_{j, k}-3 \Delta x_{j, k} / 4, y_{j, k} \pm \Delta y_{j, k} / 4\right)$ with a size of $\Delta x_{j, k} / 2 \times \Delta y_{j, k} / 2$.

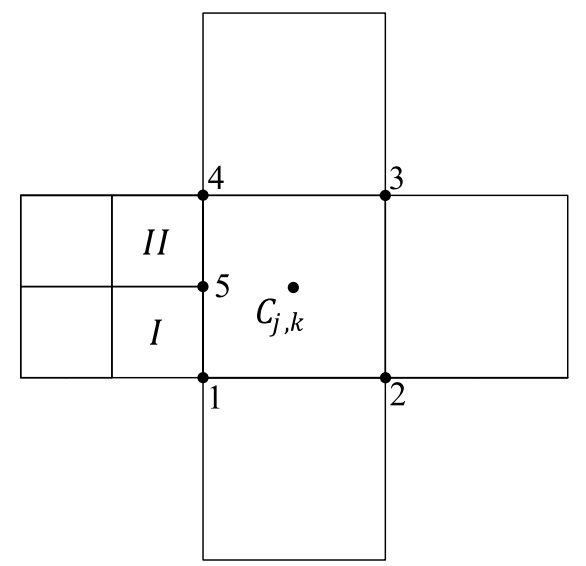

Figure 2: A configuration of cells neighboring $C_{j, k}$ in the regularised quadtree grid.

The evolution of time-dependant cell averages $\overline{\boldsymbol{U}}_{j, k}$, which are obtained after the semidiscretization of the system (3.1)-(3.4), are computed by solving the following system of Ordinary Differential Equations (ODE):

$$
\frac{\mathrm{d}}{\mathrm{d} t} \overline{\boldsymbol{U}}_{j, k}=-\frac{\boldsymbol{H}_{j+\frac{1}{2}, k}^{x}-\frac{\boldsymbol{H}_{j-\frac{1}{2}, k-\frac{1}{4}}^{x}+\boldsymbol{H}_{j-\frac{1}{2}, k+\frac{1}{4}}^{x}}{2}}{\Delta x_{j, k}}-\frac{\boldsymbol{H}_{j, k+\frac{1}{2}}^{y}-\boldsymbol{H}_{j, k-\frac{1}{2}}^{y}}{\Delta y_{j, k}}+\overline{\boldsymbol{S}}_{j, k} .
$$


In (3.6), $\boldsymbol{H}_{j+\frac{1}{2}, k}^{x}, \boldsymbol{H}_{j-\frac{1}{2}, k \pm \frac{1}{4}}^{x}, \boldsymbol{H}_{j, k+\frac{1}{2}}^{y}$ and $\boldsymbol{H}_{j, k-\frac{1}{2}}^{y}$ are the numerical fluxes, and $\overline{\boldsymbol{S}}_{j, k}$ is a cell average of the source term:

$$
\overline{\boldsymbol{S}}_{j, k} \approx \frac{1}{\Delta x_{j, k} \Delta y_{j, k}} \int_{x_{j-\frac{1}{2}}}^{x_{j+\frac{1}{2}}} \int_{y_{k-\frac{1}{2}}}^{y_{k+\frac{1}{2}}} \boldsymbol{S}(\boldsymbol{U}, B) \mathrm{d} y \mathrm{~d} x .
$$

For the sake of brevity, we have omitted all of the time-dependent indexed quantities in (3.6)-3.7).

\subsection{Piecewise bilinear reconstruction of $B$}

Cells of different sizes $\Delta x \times \Delta y, \frac{\Delta x}{2} \times \frac{\Delta y}{2}, \ldots, \frac{\Delta x}{2^{m-1}} \times \frac{\Delta y}{2^{m-1}}$ exist in the quadtree grid. The set of cells of the corresponding size is indicated by $\mathscr{C}^{(\ell)}$, that is, $\mathscr{C}^{(\ell)}=\left\{C_{j, k}:\left|C_{j, k}\right|=\frac{\Delta x}{2^{\ell-1}} \times \frac{\Delta y}{2^{\ell-1}}\right\}$. We exactly follow the steps that were introduced in [18] to reconstruct the bottom topography $\widetilde{B}(x, y)$.

\subsection{Piecewise linear reconstructions}

In this section, we construct a spatial second-order scheme, which employs a piecewise polynomial interpolation $\widetilde{\boldsymbol{U}}$, where

$$
\widetilde{\boldsymbol{U}}(x, y)=\left(\boldsymbol{U}_{x}\right)_{j, k}\left[x-x_{j}\right]+\left(\boldsymbol{U}_{y}\right)_{j, k}\left[y-y_{k}\right], \quad(x, y) \in C_{j, k} .
$$

Such a reconstruction makes it impossible to develop a well-balanced scheme. Thus, instead of reconstructing conservative variables in $\boldsymbol{U}$, we reform $\mathscr{U}:=(w, h u, h v, \rho)^{\top}$ and then obtain the point values of $\mathscr{U}$ for the cell $C_{j, k}$ in Figure 2 which results in

$$
\begin{aligned}
& \mathscr{U}_{j+\frac{1}{2}, k}^{+}=\overline{\mathscr{U}}_{j+1, k}-\frac{\Delta x_{j+1, k}}{2}\left(\mathscr{U}_{x}\right)_{j+1, k}, \quad \mathscr{U}_{j+\frac{1}{2}, k}^{-}=\overline{\mathscr{U}}_{j, k}+\frac{\Delta x_{j, k}}{2}\left(\mathscr{U}_{x}\right)_{j, k}, \\
& \mathscr{U}_{j-\frac{1}{2}, k \pm \frac{1}{4}}^{+}=\overline{\mathscr{U}}_{j, k}-\frac{\Delta x_{j, k}}{2}\left(\mathscr{U}_{x}\right)_{j, k} \pm \frac{\Delta y_{j, k}}{2}\left(\mathscr{U}_{y}\right)_{j, k}, \\
& \mathscr{U}_{j-\frac{1}{2}, k \pm \frac{1}{4}}^{-}=\overline{\mathscr{U}}_{j-\frac{1}{4}, k \pm \frac{1}{4}}+\frac{\Delta x_{j, k}}{4}\left(\mathscr{U}_{x}\right)_{j-\frac{1}{4}, k \pm \frac{1}{4}},
\end{aligned}
$$

where $\overline{\mathscr{U}}$ denote the cell averages of $\mathscr{U}$. Note that in $(3.9)$ the density variable $\rho_{j, k}$ is computed as

$$
\rho_{j, k}:=\frac{\overline{(h \rho)}_{j, k}}{\bar{h}_{j, k}}, \quad \bar{h}_{j, k}:=\bar{w}_{j, k}-B_{j, k},
$$

and the point values of $h$ and $h \rho$ read as

$$
\begin{aligned}
& h_{j-\frac{1}{2}, k \pm \frac{1}{4}}^{+}=w_{j-\frac{1}{2}, k \pm \frac{1}{4}}^{+}-B_{j-\frac{1}{2}, k \pm \frac{1}{4}}, \quad h_{j+\frac{1}{2}, k}^{-}=w_{j+\frac{1}{2}, k}^{-}-B_{j+\frac{1}{2}, k} \\
& h_{j, k-\frac{1}{2}}^{+}=w_{j, k-\frac{1}{2}}^{+}-B_{j, k-\frac{1}{2}}, \quad \text { and } \quad h_{j, k+\frac{1}{2}}^{-}=w_{j, k+\frac{1}{2}}^{-}-B_{j, k+\frac{1}{2}}
\end{aligned}
$$

and

$$
\begin{aligned}
& (h \rho)_{j-\frac{1}{2}, k \pm \frac{1}{4}}^{+}=h_{j-\frac{1}{2}, k \pm \frac{1}{4}}^{+} \cdot \rho_{j-\frac{1}{2}, k \pm \frac{1}{4}}^{+}, \quad(h \rho)_{j+\frac{1}{2}, k}^{-}=h_{j+\frac{1}{2}, k}^{-} \cdot \rho_{j+\frac{1}{2}, k}^{-}, \\
& (h \rho)_{j, k-\frac{1}{2}}^{+}=h_{j, k-\frac{1}{2}}^{+} \cdot \rho_{j, k-\frac{1}{2}}^{+}, \quad \text { and } \quad(h \rho)_{j, k+\frac{1}{2}}^{-}=h_{j, k+\frac{1}{2}}^{-} \cdot \rho_{j, k+\frac{1}{2}}^{-} \cdot
\end{aligned}
$$


We compute the slopes $\left(\mathscr{U}_{x}\right)$ and $\left(\mathscr{U}_{y}\right)$ by using the minmod limiter in order to minimize oscillations:

$$
\begin{aligned}
& \left(\mathscr{U}_{x}\right)_{j, k}=\operatorname{minmod}\left(\frac{\overline{\mathscr{U}}_{j, k}-\overline{\mathscr{U}}_{j-\frac{1}{4}, k-\frac{1}{4}}}{3 \Delta x_{j, k} / 4}, \frac{\overline{\mathscr{U}}_{j, k}-\overline{\mathscr{U}}_{j-\frac{1}{4}, k+\frac{1}{4}}}{3 \Delta x_{j, k} / 4}, \frac{\overline{\mathscr{U}}_{j+1, k}-\overline{\mathscr{U}}_{j, k}}{\Delta x_{j, k}}\right), \\
& \left(\mathscr{U}_{y}\right)_{j, k}=\operatorname{minmod}\left(\frac{\overline{\mathscr{U}}_{j, k}-\overline{\mathscr{U}}_{j, k-1}}{\Delta y_{j, k}}, \frac{\overline{\mathscr{U}}_{j, k+1}-\overline{\mathscr{U}}_{j, k}}{\Delta y_{j, k}}\right),
\end{aligned}
$$

where the minmod function is defined by

$$
\min \left\{z_{1}, z_{2}, \ldots\right\}:= \begin{cases}\min _{j}\left\{z_{j}\right\}, & \text { if } z_{j}>0 \quad \forall j \\ \max _{j}\left\{z_{j}\right\}, & \text { if } z_{j}<0 \quad \forall j \\ 0, & \text { otherwise }\end{cases}
$$

Employing the minmod limiter (3.11) guarantees the positivity of the point values of $\rho$ [15]. For the positivity-preserving correction of $w$, we exactly follow the steps in [18]. To prevent very small or even zero values of cell averages $\bar{h}$, and point values of $h$ of cell $C_{j, k}$, the corresponding point values of $u, v$, and $\rho$ are computed as follows [15, 18, 30]:

$$
u:=\frac{\sqrt{2} h(h u)}{\sqrt{h^{4}+\max \left\{h^{4}, \varepsilon\right\}}}, \quad v:=\frac{\sqrt{2} h(h v)}{\sqrt{h^{4}+\max \left\{h^{4}, \varepsilon\right\}}}, \quad \rho:=\frac{\sqrt{2} h(h \rho)}{\sqrt{h^{4}+\max \left\{h^{4}, \varepsilon\right\}}},
$$

where we choose $\varepsilon=\max \left\{\min _{j, k}\left\{\left(\Delta x_{j, k}\right)^{4}\right\}, \min _{j, k}\left\{\left(\Delta y_{j, k}\right)^{4}\right\}\right\}$. The conservative variables recalculation is done by setting:

$$
(h u):=h \cdot u, \quad(h v):=h \cdot v, \quad(h \rho):=h \cdot \rho .
$$

Note that all of the indices have been omitted in the above equations.

\subsection{Local speeds}

The one-sided local speeds of propagation, denoted at the corresponding cell interfaces by $a_{\alpha, \beta}^{ \pm}$and $b_{\gamma, \delta}^{ \pm}$can be estimated by:

$$
\begin{aligned}
& a_{\alpha, \beta}^{+}=\max \left\{u_{\alpha, \beta}^{+}+\sqrt{\frac{g}{\rho_{\circ}} h_{\alpha, \beta}^{+} \rho_{\alpha, \beta}^{+}}, u_{\alpha, \beta}^{-}+\sqrt{\frac{g}{\rho_{\circ}} h_{\alpha, \beta}^{-} \rho_{\alpha, \beta}^{-}}, 0\right\}, \\
& a_{\alpha, \beta}^{-}=\min \left\{u_{\alpha, \beta}^{+}-\sqrt{\frac{g}{\rho_{\circ}} h_{\alpha, \beta}^{+} \rho_{\alpha, \beta}^{+}}, u_{\alpha, \beta}^{-}-\sqrt{\frac{g}{\rho_{\circ}} h_{\alpha, \beta}^{-} \rho_{\alpha, \beta}^{-}}, 0\right\}, \\
& b_{\gamma, \delta}^{+}=\max \left\{v_{\gamma, \delta}^{+}+\sqrt{\frac{g}{\rho_{\circ}} h_{\gamma, \delta}^{+} \rho_{\gamma, \delta}^{+}}, v_{\gamma, \delta}^{-}+\sqrt{\frac{g}{\rho_{\circ}} h_{\gamma, \delta}^{-} \rho_{\gamma, \delta}^{-}}, 0\right\} \\
& b_{\gamma, \delta}^{-}=\min \left\{v_{\gamma, \delta}^{+}-\sqrt{\frac{g}{\rho_{\circ}} h_{\gamma, \delta}^{+} \rho_{\gamma, \delta}^{+}}, v_{\gamma, \delta}^{-}-\sqrt{\frac{g}{\rho_{\circ}} h_{\gamma, \delta}^{-} \rho_{\gamma, \delta}^{-}}, 0\right\} .
\end{aligned}
$$

where $(\alpha, \beta) \in\left\{\left(j-\frac{1}{2}, k-\frac{1}{4}\right),\left(j-\frac{1}{2}, k+\frac{1}{4}\right),\left(j+\frac{1}{2}, k\right)\right\}$ and $(\gamma, \delta) \in\left\{\left(j, k-\frac{1}{2}\right),\left(j, k+\frac{1}{2}\right)\right\}$ in Figure 2. 


\subsection{Central-upwind numerical fluxes}

We use the central-upwind fluxes from [30]:

$$
\begin{aligned}
\boldsymbol{H}_{\alpha, \beta}^{x} & =\frac{a_{\alpha, \beta}^{+} \boldsymbol{F}\left(\boldsymbol{U}_{\alpha, \beta}^{-}, B_{\alpha, \beta}\right)-a_{\alpha, \beta}^{-} \boldsymbol{F}\left(\boldsymbol{U}_{\alpha, \beta}^{+}, B_{\alpha, \beta}\right)}{a_{\alpha, \beta}^{+}-a_{\alpha, \beta}^{-}}+\frac{a_{\alpha, \beta}^{+} a_{\alpha, \beta}^{-}}{a_{\alpha, \beta}^{+}-a_{\alpha, \beta}^{-}}\left[\boldsymbol{U}_{\alpha, \beta}^{+}-\boldsymbol{U}_{\alpha, \beta}^{-}\right], \\
\boldsymbol{H}_{\gamma, \delta}^{y} & =\frac{b_{\gamma, \delta}^{+} \boldsymbol{G}\left(\boldsymbol{U}_{\gamma, \delta}^{-}, B_{\gamma, \delta}\right)-b_{\gamma, \delta}^{-} \boldsymbol{G}\left(\boldsymbol{U}_{\gamma, \delta}^{+}, B_{\gamma, \delta}\right)}{b_{\gamma, \delta}^{+}-b_{\gamma, \delta}^{-}}+\frac{b_{\gamma, \delta}^{+} b_{\gamma, \delta}^{-}}{b_{\gamma, \delta}^{+}-b_{\gamma, \delta}^{-}}\left[\boldsymbol{U}_{\gamma, \delta}^{+}-\boldsymbol{U}_{\gamma, \delta}^{-}\right] .
\end{aligned}
$$

\subsection{Well-balanced discretization of the source term}

When the discretized cell average of the source term, $\overline{\boldsymbol{S}}_{j, k}=\left(0, \bar{S}_{j, k}^{(2)}, \bar{S}_{j, k}^{(3)}, 0\right)^{\top}$, exactly balances the numerical fluxes in Equation (3.6) at the "lake-at-rest" steady state (1.2), the numerical scheme is well-balanced. This means that the right-hand side (RHS) of (3.6) vanishes as long as $\overline{\mathscr{U}}_{j, k} \equiv(\widehat{w}, 0,0, \widehat{\rho})^{\top}$ for all $(j, k)$, where $\widehat{w}$ and $\widehat{\rho}$ are constants.

Notice that at the "lake-at-rest" state, all of the reconstructed point values are $w^{ \pm}=\widetilde{w}$, $u^{ \pm}=v^{ \pm}=0$ and $\rho^{ \pm}=\widetilde{\rho}$, and thus, $a_{\alpha, \beta}^{+}=-a_{\alpha, \beta}^{-}, \forall(\alpha, \beta), b_{\gamma, \delta}^{+}=-b_{\gamma, \delta}^{-} \forall(\gamma, \delta)$, and the numerical fluxes (3.13) reduce to:

$$
\boldsymbol{H}_{\alpha, \beta}^{x}=\left(0, \frac{g}{2 \rho_{\circ}} \widehat{\rho}\left(\widehat{w}-B_{\alpha, \beta}\right)^{2}, 0,0\right)^{\top}, \quad \boldsymbol{H}_{\gamma, \delta}^{y}=\left(0,0, \frac{g}{2 \rho_{\circ}} \widehat{\rho}\left(\widehat{w}-B_{\gamma, \delta}\right)^{2}, 0\right)^{\top},
$$

and the flux terms on the RHS of $(3.6)$ then become

$$
\left.\begin{array}{l}
-\frac{\boldsymbol{H}_{j+\frac{1}{2}, k}^{x}-\frac{\boldsymbol{H}_{j-\frac{1}{2}, k-\frac{1}{4}}^{x}+\boldsymbol{H}_{j-\frac{1}{2}, k+\frac{1}{4}}^{x}}{2}-\frac{\boldsymbol{H}_{j, k+\frac{1}{2}}^{y}-\boldsymbol{H}_{j, k-\frac{1}{2}}^{y}}{\Delta y_{j, k}}}{\Delta x_{j, k}}-\frac{0}{2 \rho_{\circ}} \widehat{\rho}\left(\frac{\left(\widehat{w}-B_{j+\frac{1}{2}, k}\right)^{2}}{\Delta x_{j, k}}-\frac{\left(\widehat{w}-B_{j-\frac{1}{2}, k-\frac{1}{4}}\right)^{2}}{2 \Delta x_{j, k}}-\frac{\left(\widehat{w}-B_{j-\frac{1}{2}, k+\frac{1}{4}}\right)^{2}}{2 \Delta x_{j, k}}\right) \\
=-\frac{\left(\widehat{w}-B_{j, k+\frac{1}{2}}\right)^{2}}{\Delta y_{j, k}}-\frac{\left(\widehat{w}-B_{j, k-\frac{1}{2}}\right)^{2}}{\Delta y_{j, k}}
\end{array}\right) .
$$

By applying Green's theorem, the source term in 3.6 can be approximated by

$$
\begin{aligned}
& -\frac{g}{2 \rho_{\circ}} \rho(w-B) B_{x}=\frac{g}{2 \rho_{\circ}}\left[\rho(w-B)^{2}\right]_{x}-\frac{g}{\rho_{\circ}} \rho(w-B) w_{x}, \\
& -\frac{g}{2 \rho_{\circ}} \rho(w-B) B_{y}=\frac{g}{2 \rho_{\circ}}\left[\rho(w-B)^{2}\right]_{y}-\frac{g}{\rho_{\circ}} \rho(w-B) w_{y} .
\end{aligned}
$$

We now rewrite the cell averages of the second and third components of the integral in (3.7)

$$
\frac{g}{2 \rho_{\circ}} \int_{y_{k-\frac{1}{2}}}^{y_{k+\frac{1}{2}}}\left[\left.\rho(w-B)^{2}\right|_{x=x_{j+\frac{1}{2}}}-\left.\rho(w-B)^{2}\right|_{x=x_{j-\frac{1}{2}}}\right] \mathrm{d} y-\frac{g}{\rho_{\circ}} \int_{x_{j-\frac{1}{2}}}^{x_{j+\frac{1}{2}}} \int_{y_{k-\frac{1}{2}}}^{y_{k+\frac{1}{2}}} \rho(w-B) w_{x} \mathrm{~d} y \mathrm{~d} x
$$


and

$$
\frac{g}{2 \rho_{\circ}} \int_{x_{j-\frac{1}{2}}}^{x_{j+\frac{1}{2}}}\left[\left.\rho(w-B)^{2}\right|_{y=y_{k+\frac{1}{2}}}-\left.\rho(w-B)^{2}\right|_{y=y_{k-\frac{1}{2}}}\right] \mathrm{d} x-\frac{g}{\rho_{\circ}} \int_{x_{j-\frac{1}{2}}}^{x_{j+\frac{1}{2}}} \int_{y_{k-\frac{1}{2}}}^{y_{k+\frac{1}{2}}} \rho(w-B) w_{y} \mathrm{~d} y \mathrm{~d} x
$$

We then approximate the integrals in $(3.15)$ and $(3.16)$, which results in the following quadrature for the second and third components of the source term [18]:

$$
\begin{aligned}
& \bar{S}_{j, k}^{(2)} \approx \frac{g}{2 \rho_{\circ} \Delta x_{j, k}}\left[\rho_{j+\frac{1}{2}, k}^{-}\left(w_{j+\frac{1}{2}, k}^{-}-B_{j+\frac{1}{2}, k}\right)^{2}-\frac{\rho_{j-\frac{1}{2}, k-\frac{1}{4}}^{+}\left(w_{j-\frac{1}{2}, k-\frac{1}{4}}^{+}-B_{j-\frac{1}{2}, k-\frac{1}{4}}\right)^{2}}{2}\right. \\
& \left.-\frac{\rho_{j-\frac{1}{2}, k+\frac{1}{4}}^{+}\left(w_{j-\frac{1}{2}, k+\frac{1}{4}}^{+}-B_{j-\frac{1}{2}, k+\frac{1}{4}}\right)^{2}}{2}\right]-\frac{g}{\rho_{\circ}} \rho_{j, k}\left(w_{x}\right)_{j, k}\left(\bar{w}_{j, k}-B_{j, k}\right), \\
& \bar{S}_{j, k}^{(3)} \approx \frac{g}{2 \rho_{\circ} \Delta y_{j, k}}\left[\rho_{j, k+\frac{1}{2}}^{-}\left(w_{j, k+\frac{1}{2}}^{-}-B_{j, k+\frac{1}{2}}\right)^{2}-\rho_{j, k-\frac{1}{2}}^{+}\left(w_{j, k-\frac{1}{2}}^{+}-B_{j, k-\frac{1}{2}}\right)^{2}\right] \\
& -\frac{g}{\rho_{\circ}} \rho_{j, k}\left(w_{y}\right)_{j, k}\left(\bar{w}_{j, k}-B_{j, k}\right) \text {. }
\end{aligned}
$$

We finally state that the scheme now preserves the solution at "lake-at-rest" where, $\left(w_{x}\right)_{j, k}=$ $\left(w_{y}\right)_{j, k} \equiv 0, \forall(j, k)$ and thus (3.14) and (3.17) express that the RHS of (3.6) vanishes and therefore, the scheme is well-balanced.

\subsection{Positivity-preserving property}

In this section, we extend the positivity-preserving proof from [18] to implement on the coupled variable density system. We use a forward Euler method to integrate Equation (3.6) in time, which results in

$$
\begin{gathered}
\bar{w}_{j, k}^{n+1}=\bar{w}_{j, k}^{n}-\lambda_{j, k}^{n}\left(H_{j+\frac{1}{2}, k}^{x,(1)}-\frac{H_{j-\frac{1}{2}, k-\frac{1}{4}}^{x,(1)}+H_{j-\frac{1}{2}, k+\frac{1}{4}}^{x,(1)}}{2}\right)-\mu_{j, k}^{n}\left(H_{j, k+\frac{1}{2}}^{y,(1)}-H_{j, k-\frac{1}{2}}^{y,(1)}\right), \\
\overline{(h \rho)}_{j, k}^{n+1}=\overline{(h \rho)}_{j, k}^{n}-\lambda_{j, k}^{n}\left(H_{j+\frac{1}{2}, k}^{x,(4)}-\frac{H_{j-\frac{1}{2}, k-\frac{1}{4}}^{x,(4)}+H_{j-\frac{1}{2}, k+\frac{1}{4}}^{x,(4)}}{2}\right)-\mu_{j, k}^{n}\left(H_{j, k+\frac{1}{2}}^{y,(4)}-H_{j, k-\frac{1}{2}}^{y,(4)}\right),
\end{gathered}
$$

where $\bar{w}_{j, k}^{n}:=\bar{w}_{j, k}\left(t^{n}\right), \bar{w}_{j, k}^{n+1}:=\bar{w}_{j, k}\left(t^{n+1}\right),{\overline{(h \rho)_{j, k}}}_{j}^{n}:=\overline{(h \rho)}_{j, k}\left(t^{n}\right)$, and $\overline{(h \rho)}_{j, k}^{n+1}:=\overline{(h \rho)}_{j, k}\left(t^{n+1}\right)$ with $t^{n+1}=t^{n}+\Delta t^{n}, \lambda_{j, k}^{n}:=\Delta t^{n} / \Delta x_{j, k}, \mu_{j, k}^{n}:=\Delta t^{n} / \Delta y_{j, k}$, and the numerical fluxes on the RHS are evaluated at time level $t=t^{n}$ using (3.13):

$$
\begin{aligned}
& H_{\alpha, \beta}^{x,(1)}=\frac{a_{\alpha, \beta}^{+}(h u)_{\alpha, \beta}^{-}-a_{\alpha, \beta}^{-}(h u)_{\alpha, \beta}^{+}}{a_{\alpha, \beta}^{+}-a_{\alpha, \beta}^{-}}+\frac{a_{\alpha, \beta}^{+} a_{\alpha, \beta}^{-}}{a_{\alpha, \beta}^{+}-a_{\alpha, \beta}^{-}}\left[w_{\alpha, \beta}^{+}-w_{\alpha, \beta}^{-}\right], \\
& H_{\gamma, \delta}^{y,(1)}=\frac{b_{\gamma, \delta}^{+}(h v)_{\gamma, \delta}^{-}-b_{\gamma, \delta}^{-}(h v)_{\gamma, \delta}^{+}}{b_{\gamma, \delta}^{+}-b_{\gamma, \delta}^{-}}+\frac{b_{\gamma, \delta}^{+} b_{\gamma, \delta}^{-}}{b_{\gamma, \delta}^{+}-b_{\gamma, \delta}^{-}}\left[w_{\gamma, \delta}^{+}-w_{\gamma, \delta}^{-}\right],
\end{aligned}
$$


and

$$
\begin{aligned}
& H_{\alpha, \beta}^{x,(4)}=\frac{a_{\alpha, \beta}^{+} \rho_{\alpha, \beta}^{-}(h u)_{\alpha, \beta}^{-}-a_{\alpha, \beta}^{-} \rho_{\alpha, \beta}^{+}(h u)_{\alpha, \beta}^{+}}{a_{\alpha, \beta}^{+}-a_{\alpha, \beta}^{-}}+\frac{a_{\alpha, \beta}^{+} a_{\alpha, \beta}^{-}}{a_{\alpha, \beta}^{+}-a_{\alpha, \beta}^{-}}\left[(h \rho)_{\alpha, \beta}^{+}-(h \rho)_{\alpha, \beta}^{-}\right], \\
& H_{\gamma, \delta}^{y,(4)}=\frac{b_{\gamma, \delta}^{+} \rho_{\gamma, \delta}^{-}(h v)_{\gamma, \delta}^{-}-b_{\gamma, \delta}^{-} \rho_{\gamma, \delta}^{+}(h v)_{\gamma, \delta}^{+}}{b_{\gamma, \delta}^{+}-b_{\gamma, \delta}^{-}}+\frac{b_{\gamma, \delta}^{+} b_{\gamma, \delta}^{-}}{b_{\gamma, \delta}^{+}-b_{\gamma, \delta}^{-}}\left[(h \rho)_{\gamma, \delta}^{+}-(h \rho)_{\gamma, \delta}^{-}\right] .
\end{aligned}
$$

If $\bar{h}_{j, k}^{n} \geq 0$ for all $(j, k)$, then the point values of the computed $h$ are nonnegative [30]. Moreover, using the bilinear piece for the bottom topography from [18] and the similar relationships for the reconstructed point values of $w$, we have

$$
\bar{h}_{j, k}^{n}=\frac{1}{4}\left(\frac{h_{j-\frac{1}{2}, k-\frac{1}{4}}^{+}+h_{j-\frac{1}{2}, k+\frac{1}{4}}^{+}}{2}+h_{j+\frac{1}{2}, k}^{-}+h_{j, k-\frac{1}{2}}^{+}+h_{j, k+\frac{1}{2}}^{-}\right)
$$

for the grid configuration in Figure 2.

We now subtract $B_{j, k}$ from both sides of $(3.18)$ and use $(3.20)$ and $(3.22)$ to rewrite $(3.18)$ as follows:

$$
\begin{aligned}
& \bar{h}_{j, k}^{n+1}=-\lambda_{j, k}^{n} a_{j+\frac{1}{2}, k}^{-} \cdot \frac{a_{j+\frac{1}{2}, k}^{+}-u_{j+\frac{1}{2}, k}^{+}}{a_{j+\frac{1}{2}, k}^{+}-a_{j+\frac{1}{2}, k}^{-}} \cdot h_{j+\frac{1}{2}, k}^{+}+\left[\frac{1}{4}-\lambda_{j, k}^{n} a_{j+\frac{1}{2}, k}^{+} \cdot \frac{u_{j+\frac{1}{2}, k}^{-}-a_{j+\frac{1}{2}, k}^{-}}{a_{j+\frac{1}{2}, k}^{+}-a_{j+\frac{1}{2}, k}^{-}}\right] h_{j+\frac{1}{2}, k}^{-} \\
& +\frac{\lambda_{j, k}^{n} a_{j-\frac{1}{2}, k-\frac{1}{4}}^{+}}{2} \cdot \frac{u_{j-\frac{1}{2}, k-\frac{1}{4}}^{-}-a_{j-\frac{1}{2}, k-\frac{1}{4}}^{-}}{a_{j-\frac{1}{2}, k-\frac{1}{4}}^{+}-a_{j-\frac{1}{2}, k-\frac{1}{4}}^{-}} \cdot h_{j-\frac{1}{2}, k-\frac{1}{4}}^{+} \\
& +\frac{1}{2}\left[\frac{1}{4}-\lambda_{j, k}^{n} a_{j-\frac{1}{2}, k-\frac{1}{4}}^{-} \cdot \frac{a_{j-\frac{1}{2}, k-\frac{1}{4}}^{+}-u_{j-\frac{1}{2}, k-\frac{1}{4}}^{+}}{a_{j-\frac{1}{2}, k-\frac{1}{4}}^{+}-a_{j-\frac{1}{2}, k-\frac{1}{4}}^{-}}\right] h_{j-\frac{1}{2}, k-\frac{1}{4}}^{+} \\
& +\frac{\lambda_{j, k}^{n} a_{j-\frac{1}{2}, k+\frac{1}{4}}^{+}}{2} \cdot \frac{u_{j-\frac{1}{2}, k+\frac{1}{4}}^{-}-a_{j-\frac{1}{2}, k+\frac{1}{4}}^{-}}{a_{j-\frac{1}{2}, k+\frac{1}{4}}^{+}-a_{j-\frac{1}{2}, k+\frac{1}{4}}^{-}} \cdot h_{j-\frac{1}{2}, k+\frac{1}{4}}^{+} \\
& +\frac{1}{2}\left[\frac{1}{4}-\lambda_{j, k}^{n} a_{j-\frac{1}{2}, k+\frac{1}{4}}^{-} \cdot \frac{a_{j-\frac{1}{2}, k+\frac{1}{4}}^{+}-u_{j-\frac{1}{2}, k+\frac{1}{4}}^{+}}{a_{j-\frac{1}{2}, k+\frac{1}{4}}^{+}-a_{j-\frac{1}{2}, k+\frac{1}{4}}^{-}}\right] h_{j-\frac{1}{2}, k+\frac{1}{4}}^{+} \\
& -\mu_{j, k}^{n} b_{j, k+\frac{1}{2}}^{-} \cdot \frac{b_{j, k+\frac{1}{2}}^{+}-v_{j, k+\frac{1}{2}}^{+}}{b_{j, k+\frac{1}{2}}^{+}-b_{j, k+\frac{1}{2}}^{-}} \cdot h_{j, k+\frac{1}{2}}^{+}+\left[\frac{1}{4}-\mu_{j, k}^{n} b_{j, k+\frac{1}{2}}^{+} \cdot \frac{v_{j, k+\frac{1}{2}}^{-}-b_{j, k+\frac{1}{2}}^{-}}{b_{j, k+\frac{1}{2}}^{+}-b_{j, k+\frac{1}{2}}^{-}}\right] h_{j, k+\frac{1}{2}}^{-} \\
& +\mu_{j, k}^{n} b_{j, k-\frac{1}{2}}^{+} \cdot \frac{v_{j, k-\frac{1}{2}}^{-}-b_{j, k-\frac{1}{2}}^{-}}{b_{j, k-\frac{1}{2}}^{+}-b_{j, k-\frac{1}{2}}^{-}} \cdot h_{j, k-\frac{1}{2}}^{-}+\left[\frac{1}{4}+\mu_{j, k}^{n} b_{j, k-\frac{1}{2}}^{-} \cdot \frac{b_{j, k-\frac{1}{2}}^{+}-v_{j, k-\frac{1}{2}}^{+}}{b_{j, k-\frac{1}{2}}^{+}-b_{j, k-\frac{1}{2}}^{-}}\right] h_{j, k-\frac{1}{2}}^{+} \text {. }
\end{aligned}
$$

This shows that the cell averages of $h$ are linear combinations of the reconstructed nonnegative point values of $h$. Thus, $\bar{h}_{j, k}^{n+1} \geq 0$ provided all of the coefficients in this linear combination are nonnegative.

One can obtain a similar proof for positivity of $\overline{(h \rho)}_{j, k}^{n+1}$ by using the following statements 
[15],

$$
\bar{h}_{j, k}=\frac{h_{j+\frac{1}{2}, k}^{-}+\frac{h_{j-\frac{1}{2}, k-\frac{1}{4}}^{+}+h_{j-\frac{1}{2}, k+\frac{1}{4}}^{+}}{2}}{2}, \quad \rho_{j, k}=\frac{\rho_{j+\frac{1}{2}, k}^{-}+\frac{\rho_{j-\frac{1}{2}, k-\frac{1}{4}}^{+}+\rho_{j-\frac{1}{2}, k+\frac{1}{4}}^{+}}{2}}{2},
$$

and thereby, utilizing (3.10), one obtains:

$$
\begin{aligned}
\overline{(h \rho)_{j, k}^{n}=} & \frac{1}{4}\left[h_{j+\frac{1}{2}, k}^{-} \rho_{j+\frac{1}{2}, k}^{-}+\frac{h_{j-\frac{1}{2}, k-\frac{1}{4}}^{+} \rho_{j-\frac{1}{2}, k-\frac{1}{4}}^{+}+h_{j-\frac{1}{2}, k+\frac{1}{4}}^{+} \rho_{j-\frac{1}{2}, k+\frac{1}{4}}^{+}}{2}\right] \\
& +\frac{1}{4}\left[\frac{h_{j+\frac{1}{2}, k}^{-} \rho_{j-\frac{1}{2}, k-\frac{1}{4}}^{+}+h_{j+\frac{1}{2}, k}^{-} \rho_{j-\frac{1}{2}, k+\frac{1}{4}}^{+}+h_{j-\frac{1}{2}, k-\frac{1}{4}}^{+} \rho_{j+\frac{1}{2}, k}^{-}+h_{j-\frac{1}{2}, k+\frac{1}{4}}^{+} \rho_{j+\frac{1}{2}, k}^{-}}{2}\right] \\
& +\frac{1}{4}\left[\frac{h_{j-\frac{1}{2}, k-\frac{1}{4}}^{+} \rho_{j-\frac{1}{2}, k+\frac{1}{4}}^{+}+h_{j-\frac{1}{2}, k+\frac{1}{4}}^{+} \rho_{j-\frac{1}{2}, k-\frac{1}{4}}^{+}}{4}\right] .
\end{aligned}
$$

Similarly, it can be shown that

$$
\overline{(h \rho)}_{j, k}^{n}=\frac{1}{4}\left[h_{j, k+\frac{1}{2}}^{-} \rho_{j, k+\frac{1}{2}}^{-}+h_{j, k-\frac{1}{2}}^{+} \rho_{j, k-\frac{1}{2}}^{+}\right]+\frac{1}{4}\left[h_{j, k+\frac{1}{2}}^{-} \rho_{j, k-\frac{1}{2}}^{+}+h_{j, k-\frac{1}{2}}^{+} \rho_{j, k+\frac{1}{2}}^{-}\right] .
$$

Finally, from (3.25) and (3.26) we have

$$
\begin{aligned}
\overline{(h \rho)}_{j, k}^{n}= & \frac{1}{8}\left[h_{j+\frac{1}{2}, k}^{-} \rho_{j+\frac{1}{2}, k}^{-}+h_{j, k+\frac{1}{2}}^{-} \rho_{j, k+\frac{1}{2}}^{-}+h_{j, k-\frac{1}{2}}^{+} \rho_{j, k-\frac{1}{2}}^{+}\right] \\
& +\frac{1}{8}\left[\frac{h_{j-\frac{1}{2}, k-\frac{1}{4}}^{+} \rho_{j-\frac{1}{2}, k-\frac{1}{4}}^{+}+h_{j-\frac{1}{2}, k+\frac{1}{4}}^{+} \rho_{j-\frac{1}{2}, k+\frac{1}{4}}^{+}}{2}\right] \\
& +\frac{1}{8}\left[\frac{h_{j+\frac{1}{2}, k}^{-} \rho_{j-\frac{1}{2}, k-\frac{1}{4}}^{+}+h_{j+\frac{1}{2}, k}^{-} \rho_{j-\frac{1}{2}, k+\frac{1}{4}}^{+}+h_{j-\frac{1}{2}, k-\frac{1}{4}}^{+} \rho_{j+\frac{1}{2}, k}^{-}+h_{j-\frac{1}{2}, k+\frac{1}{4}}^{+} \rho_{j+\frac{1}{2}, k}^{-}}{2}\right] \\
& +\frac{1}{8}\left[\frac{h_{j-\frac{1}{2}, k-\frac{1}{4}}^{+} \rho_{j-\frac{1}{2}, k+\frac{1}{4}}^{+}+h_{j-\frac{1}{2}, k+\frac{1}{4}}^{+} \rho_{j-\frac{1}{2}, k-\frac{1}{4}}^{+}}{4}\right] .
\end{aligned}
$$


We rewrite $(3.19)$ as follows:

$$
\begin{aligned}
& \overline{(h \rho)}_{j, k}^{n+1}=-\lambda_{j, k}^{n} a_{j+\frac{1}{2}, k}^{-} \cdot \frac{a_{j+\frac{1}{2}, k}^{+}-u_{j+\frac{1}{2}, k}^{+}}{a_{j+\frac{1}{2}, k}^{+}-a_{j+\frac{1}{2}, k}^{-}} \cdot h_{j+\frac{1}{2}, k}^{+} \rho_{j+\frac{1}{2}, k}^{+} \\
& +\left[\frac{1}{8}-\lambda_{j, k}^{n} a_{j+\frac{1}{2}, k}^{+} \cdot \frac{u_{j+\frac{1}{2}, k}^{-}-a_{j+\frac{1}{2}, k}^{-}}{a_{j+\frac{1}{2}, k}^{+}-a_{j+\frac{1}{2}, k}^{-}}\right] h_{j+\frac{1}{2}, k}^{-} \rho_{j+\frac{1}{2}, k}^{-} \\
& +\frac{\lambda_{j, k}^{n} a_{j-\frac{1}{2}, k-\frac{1}{4}}^{+}}{2} \cdot \frac{u_{j-\frac{1}{2}, k-\frac{1}{4}}^{-}-a_{j-\frac{1}{2}, k-\frac{1}{4}}^{-}}{a_{j-\frac{1}{2}, k-\frac{1}{4}}^{+}-a_{j-\frac{1}{2}, k-\frac{1}{4}}^{-}} \cdot h_{j-\frac{1}{2}, k-\frac{1}{4}}^{+} \rho_{j-\frac{1}{2}, k-\frac{1}{4}}^{+} \\
& +\frac{1}{2}\left[\frac{1}{8}-\lambda_{j, k}^{n} a_{j-\frac{1}{2}, k-\frac{1}{4}}^{-} \cdot \frac{a_{j-\frac{1}{2}, k-\frac{1}{4}}^{+}-u_{j-\frac{1}{2}, k-\frac{1}{4}}^{+}}{a_{j-\frac{1}{2}, k-\frac{1}{4}}^{+}-a_{j-\frac{1}{2}, k-\frac{1}{4}}^{-}}\right] h_{j-\frac{1}{2}, k-\frac{1}{4}}^{+} \rho_{j-\frac{1}{2}, k-\frac{1}{4}}^{+} \\
& +\frac{\lambda_{j, k}^{n} a_{j-\frac{1}{2}, k+\frac{1}{4}}^{+}}{2} \cdot \frac{u_{j-\frac{1}{2}, k+\frac{1}{4}}^{-}-a_{j-\frac{1}{2}, k+\frac{1}{4}}^{-}}{a_{j-\frac{1}{2}, k+\frac{1}{4}}^{+}-a_{j-\frac{1}{2}, k+\frac{1}{4}}^{-}} \cdot h_{j-\frac{1}{2}, k+\frac{1}{4}}^{+} \rho_{j-\frac{1}{2}, k+\frac{1}{4}}^{+} \\
& +\frac{1}{2}\left[\frac{1}{8}-\lambda_{j, k}^{n} a_{j-\frac{1}{2}, k+\frac{1}{4}}^{-} \cdot \frac{a_{j-\frac{1}{2}, k+\frac{1}{4}}^{+}-u_{j-\frac{1}{2}, k+\frac{1}{4}}^{+}}{a_{j-\frac{1}{2}, k+\frac{1}{4}}^{+}-a_{j-\frac{1}{2}, k+\frac{1}{4}}^{-}}\right] h_{j-\frac{1}{2}, k+\frac{1}{4}}^{+} \rho_{j-\frac{1}{2}, k+\frac{1}{4}}^{+} \\
& -\mu_{j, k}^{n} b_{j, k+\frac{1}{2}}^{-} \cdot \frac{b_{j, k+\frac{1}{2}}^{+}-v_{j, k+\frac{1}{2}}^{+}}{b_{j, k+\frac{1}{2}}^{+}-b_{j, k+\frac{1}{2}}^{-}} \cdot h_{j, k+\frac{1}{2}}^{+} \rho_{j, k+\frac{1}{2}}^{+} \\
& +\left[\frac{1}{8}-\mu_{j, k}^{n} b_{j, k+\frac{1}{2}}^{+} \cdot \frac{v_{j, k+\frac{1}{2}}^{-}-b_{j, k+\frac{1}{2}}^{-}}{b_{j, k+\frac{1}{2}}^{+}-b_{j, k+\frac{1}{2}}^{-}}\right] h_{j, k+\frac{1}{2}}^{-} \rho_{j, k+\frac{1}{2}}^{-} \\
& +\mu_{j, k}^{n} b_{j, k-\frac{1}{2}}^{+} \cdot \frac{v_{j, k-\frac{1}{2}}^{-}-b_{j, k-\frac{1}{2}}^{-}}{b_{j, k-\frac{1}{2}}^{+}-b_{j, k-\frac{1}{2}}^{-}} \cdot h_{j, k-\frac{1}{2}}^{-} \rho_{j, k-\frac{1}{2}}^{-} \\
& +\left[\frac{1}{8}+\mu_{j, k}^{n} b_{j, k-\frac{1}{2}}^{-} \cdot \frac{b_{j, k-\frac{1}{2}}^{+}-v_{j, k-\frac{1}{2}}^{+}}{b_{j, k-\frac{1}{2}}^{+}-b_{j, k-\frac{1}{2}}^{-}}\right] h_{j, k-\frac{1}{2}}^{+} \rho_{j, k-\frac{1}{2}}^{+} \\
& +\frac{1}{8}\left[\frac{h_{j+\frac{1}{2}, k}^{-} \rho_{j-\frac{1}{2}, k-\frac{1}{4}}^{+}+h_{j+\frac{1}{2}, k}^{-} \rho_{j-\frac{1}{2}, k+\frac{1}{4}}^{+}+h_{j-\frac{1}{2}, k-\frac{1}{4}}^{+} \rho_{j+\frac{1}{2}, k}^{-}+h_{j-\frac{1}{2}, k+\frac{1}{4}}^{+} \rho_{j+\frac{1}{2}, k}^{-}}{2}\right] \\
& +\frac{1}{8}\left[\frac{h_{j-\frac{1}{2}, k-\frac{1}{4}}^{+} \rho_{j-\frac{1}{2}, k+\frac{1}{4}}^{+}+h_{j-\frac{1}{2}, k+\frac{1}{4}}^{+} \rho_{j-\frac{1}{2}, k-\frac{1}{4}}^{+}}{4}\right] .
\end{aligned}
$$

From (3.9) and positivity-preserving correction of $w$, which guarantee the positivity of the reconstructed point values of $h$, the last two terms in (3.28) are nonnegative. The other terms are also nonnegative similar to those in (3.23). Note that using the minmod limiter (3.11) guarantees positivity of the point values of $\rho$. Since both $h_{j, k}^{n+1}$ and $\overline{(h \rho)}_{j, k}^{n+1}$ are nonnegative, thus $\rho_{j, k}^{n+1}=\overline{(h \rho)}_{j, k}^{n+1} / h_{j, k}^{n+1}$ is nonnegative as well. 
We use (3.12) to satisfy the Courant-Friedrichs-Lewy (CFL) type condition as follows:

$$
\Delta t \leq \frac{1}{8} \min \left[\min _{j, k}\left\{\frac{\Delta x_{j, k}}{\max _{(\alpha, \beta)}\left[\max \left\{a_{\alpha, \beta}^{+},-a_{\alpha, \beta}^{-}\right\}\right]}\right\}, \min _{j, k}\left\{\frac{\Delta y_{j, k}}{\max _{(\gamma, \delta)}\left[\max \left\{b_{\gamma, \delta}^{+},-b_{\gamma, \delta}^{-}\right\}\right]}\right\}\right] .
$$

Finally, in all of the numerical experiments, we have used the three-stage third-order strong stability preserving (SSP) Runge-Kutta solver (see, e.g., [18, 19, 20]).

\subsection{Quadtree grid adaptivity}

At the new time level $t=t^{n+1}$, the quadtree grid locally refines or coarsens for the next timestep. We first need to compute the slopes $\left\{\left(w_{x}\right)_{j, k}^{n+1}\right\}$ and $\left\{\left(w_{y}\right)_{j, k}^{n+1}\right\}$, and $\left\{\left(\rho_{x}\right)_{j, k}^{n+1}\right\}$ and $\left\{\left(\rho_{y}\right)_{j, k}^{n+1}\right\}$ on the old grid (which is denoted by $\left\{C_{j, k}^{\text {old }}\right\}$ ) according to $\$ 3.3$ and then select the centers of those cells $C_{j, k}^{\text {old }}$, at which (see [18]):

$$
\left(w_{x}\right)_{j, k}^{n+1} \geq C_{w, \text { seed }} \quad \text { or } \quad\left(w_{y}\right)_{j, k}^{n+1} \geq C_{w, \text { seed }}
$$

and

$$
\left(\rho_{x}\right)_{j, k}^{n+1} \geq C_{\rho, \text { seed }} \quad \text { or } \quad\left(\rho_{y}\right)_{j, k}^{n+1} \geq C_{\rho, \text { seed }} .
$$

We denote the required seeding points to generate the new grid by $\left\{C_{j, k}^{\text {new }}\right\}$. In 3.29 and (3.30), $C_{w \text {, seed }}$ and $C_{\rho \text {, seed }}$ are constants that depend on the problem at hand (e.g. the maximum level of the quadtree, the Froude number, and the bottom topography function). When the grid locally refines or coarsens, at the end of the evolution step, the solution in terms of the computed cell averages $\left\{\left(\overline{\mathscr{U}}_{j, k}^{n+1}\right)_{\text {old }}\right\}$ over the grid $\left\{C_{j, k}^{\text {old }}\right\}$, should be projected onto the new grid $\left\{C_{j, k}^{\text {new }}\right\}$. This should be done in a conservative manner as follows:

Case 1: If $C_{j, k}^{\text {new }}=C_{j^{\prime}, k^{\prime}}^{\text {old }}$ for some $\left(j^{\prime}, k^{\prime}\right)$, that is, if the cell $C_{j^{\prime}, k^{\prime}}^{\text {old }}$ does not need to be refined/coarsened, then

$$
\left(\overline{\mathscr{U}}_{j, k}^{n+1}\right)_{\text {new }}=\left(\overline{\mathscr{U}}_{j^{\prime}, k^{\prime}}^{n+1}\right)_{\text {old }} .
$$

Case 2: If $C_{j, k}^{\text {new }} \in \mathscr{C}^{\ell+p}$ is a "child" cell of $C_{j^{\prime}, k^{\prime}}^{\text {old }} \in \mathscr{C}^{\ell}$ for some $j^{\prime}, k^{\prime}$ and $p>0$ (that is, if the cell $C_{j^{\prime}, k^{\prime}}^{\text {old }}$ was refined and $\left.C_{j, k}^{\text {new }} \subset C_{j^{\prime}, k^{\prime}}^{\text {old }}\right)$, then

$$
\left(\overline{\mathscr{U}}_{j, k}^{n+1}\right)_{\text {new }}=\left(\overline{\mathscr{U}}_{j^{\prime}, k^{\prime}}^{n+1}\right)_{\text {old }}+\left(\left(\mathscr{U}_{x}\right)_{j^{\prime}, k^{\prime}}^{n+1}\right)_{\text {old }}\left[x_{j}^{\text {new }}-x_{j^{\prime}}^{\text {old }}\right]+\left(\left(\mathscr{U}_{y}\right)_{j^{\prime}, k^{\prime}}^{n+1}\right)_{\text {old }}\left[y_{k}^{\text {new }}-y_{k^{\prime}}^{\text {old }}\right] .
$$

Case 3: If $C_{j, k}^{\text {new }} \in \mathscr{C}^{\ell-p}$ is a "parent" cell of $C_{j^{\prime}, k^{\prime}}^{\text {old }} \in \mathscr{C}^{\ell}$ for some $j^{\prime}, k^{\prime}$ and $p>0$ (that is, if the cell $C_{j^{\prime}, k^{\prime}}^{\text {old }}$ was coarsened and $\left.C_{j, k}^{\text {new }} \supset C_{j^{\prime}, k^{\prime}}^{\text {old }}\right)$, then

$$
\left(\overline{\mathscr{U}}_{j, k}^{n+1}\right)_{\text {new }}=\frac{1}{4^{p}} \sum_{j^{\prime \prime}, k^{\prime \prime}: C_{j^{\prime \prime}, k^{\prime \prime}}^{\text {old }} \subset C_{j, k}^{\text {new }}}\left(\overline{\mathscr{U}}_{j^{\prime \prime}, k^{\prime \prime}}^{n+1}\right)_{\text {old }} .
$$

\section{Numerical examples}

In this section, we investigate the performance of the proposed scheme in four numerical examples. In all of the examples, we take $g=1$ and $\rho_{\circ}=997$. 


\section{Example 1 - Circular dam break with constant density}

We demonstrate the ability of the proposed scheme to generate adaptive grids at each timestep and maintain symmetry in this example. A circular water column collapses on a horizontal flat bottom topography $40 \times 40$ dimensions plane [16, 24] where:

$$
\begin{aligned}
& w(x, y, 0)= \begin{cases}2, & (x-20)^{2}+(y-20)^{2}<2.5^{2}, \\
1, & \text { otherwise },\end{cases} \\
& u(x, y, 0)=v(x, y, 0) \equiv 0, \quad \rho(x, y, 0) \equiv \rho_{\circ} .
\end{aligned}
$$

Furthermore, we take $m=9$ refinement levels of the quadtree grid and set $C_{w \text {, seed }}=5 \times 10^{-4}$ in (3.29). The solution runs until the final time $t=4$. Water surface contours and the respective quadtree grids are illustrated in Figure 3. The quadtree grid starts with 2,134 cells and ends with 35,200 cells at $t=4$. The results in Figure 3 show that the solution follows the same evolution in comparison with the ones obtained in [16, 24].

\section{Example 2-Dam break with density discontinuity over a hump}

This example is based on the benchmark in [12, 24]. We show the capability of the centralupwind quadtree scheme to maintain the well-balanced property, symmetry, and to generate adaptive grids. We consider the computational domain to be $[0,2] \times[0,1]$ with the following initial conditions:

$$
\rho(x, y, 0)=\left\{\begin{array}{ll}
997, & x<1, \\
1200, & x \geq 1,
\end{array} \quad u(x, y, 0)=v(x, y, 0) \equiv 0, \quad w(x, y, 0) \equiv 1,\right.
$$

and the given bottom topography

$$
B(x, y)=0.8 e^{-5(x-0.9)^{2}-50(y-0.5)^{2}} .
$$

We set $C_{w \text {, seed }}=10^{-2}$ and $C_{\rho \text {, seed }}=10$ in (3.29) and (3.30), and $m=8$. A solid wall boundary condition is used at the top and bottom boundaries. For the sake of simplicity, we set the left and the right boundaries to Dirichlet boundary conditions. We run the solution up to the final time $t=0.8$ with the non-well-balanced and well-balanced schemes. For the non-well-balanced scheme, the source approximations read as:

$$
\begin{aligned}
& \bar{S}_{j, k}^{(2)}=-\frac{g \overline{(h \rho)}_{j, k}}{\rho_{\circ} \Delta x_{j, k}}\left[\frac{B_{j+\frac{1}{2}, k+\frac{1}{2}}+B_{j+\frac{1}{2}, k-\frac{1}{2}}}{2}-\frac{B_{j-\frac{1}{2}, k+\frac{1}{2}}+B_{j-\frac{1}{2}, k-\frac{1}{2}}}{2}\right], \\
& \bar{S}_{j, k}^{(3)}=-\frac{g \overline{(h \rho)}_{j, k}}{\rho_{\circ} \Delta x_{j, k}}\left[\frac{B_{j+\frac{1}{2}, k+\frac{1}{2}}+B_{j+\frac{1}{2}, k-\frac{1}{2}}}{2}-\frac{B_{j-\frac{1}{2}, k+\frac{1}{2}}+B_{j-\frac{1}{2}, k-\frac{1}{2}}}{2}\right] .
\end{aligned}
$$

Water surface contours and the respective quadtree grids of the solution of the non-wellbalanced scheme are demonstrated in Figure 4. The quadtree grid starts with 1,184 cells and ends with 27,749 cells at $t=0.8$. The well-balanced solution is presented in Figure 5 where the quadtree has a minimum of 1,184 and a maximum of 18,497 cells. Utilizing the well-balanced scheme reduces the number of cells in the quadtree grid; thereby, the computational cost is reduced and unphysical oscillations are eliminated. 

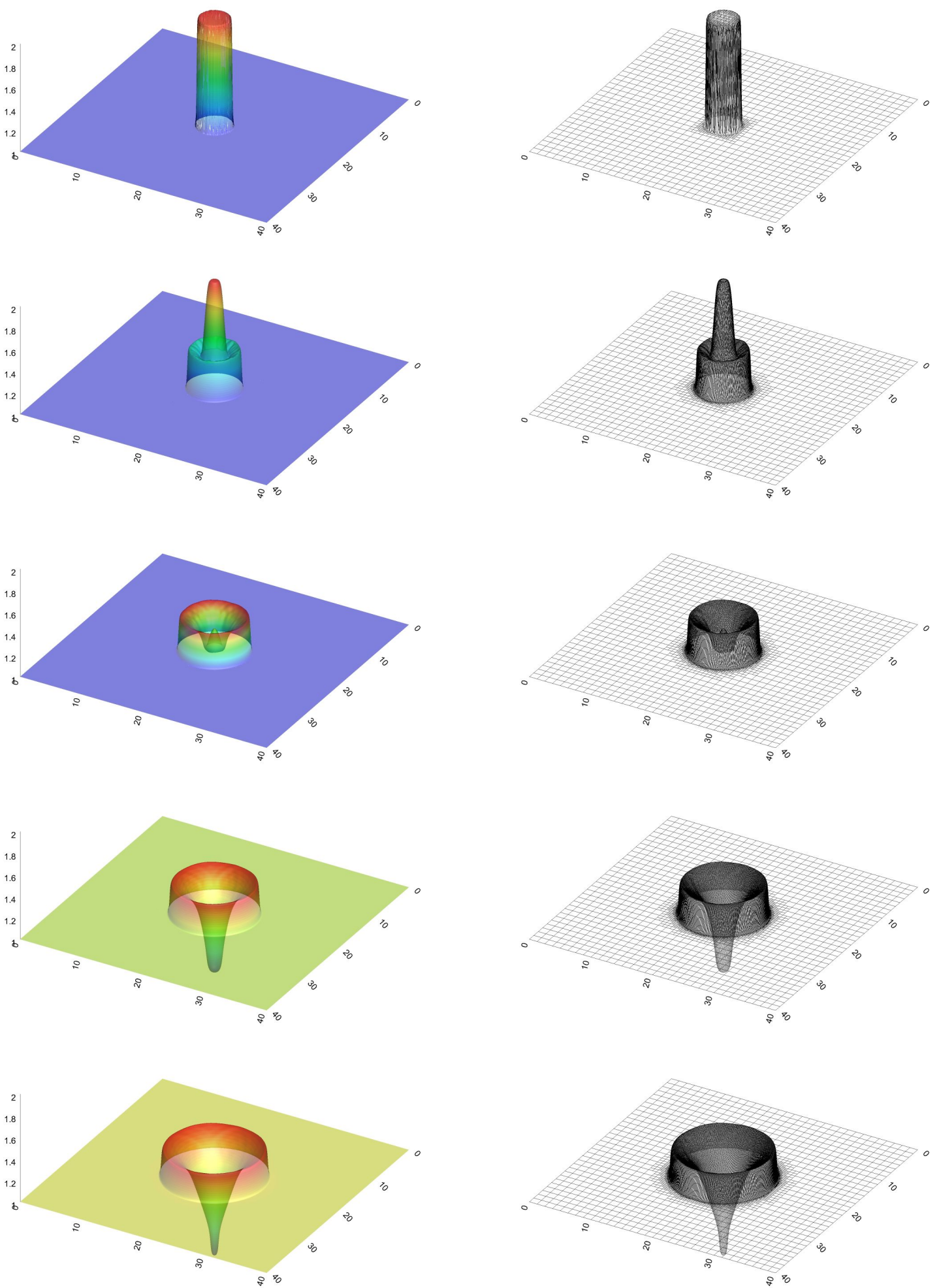

Figure 3: Example 1: Initial and computed water surface $w(x, y, t)$ (left column) and corresponding quadtree grids (right column) for $t=0,1,2,3$, and 4 (from top to bottom). 

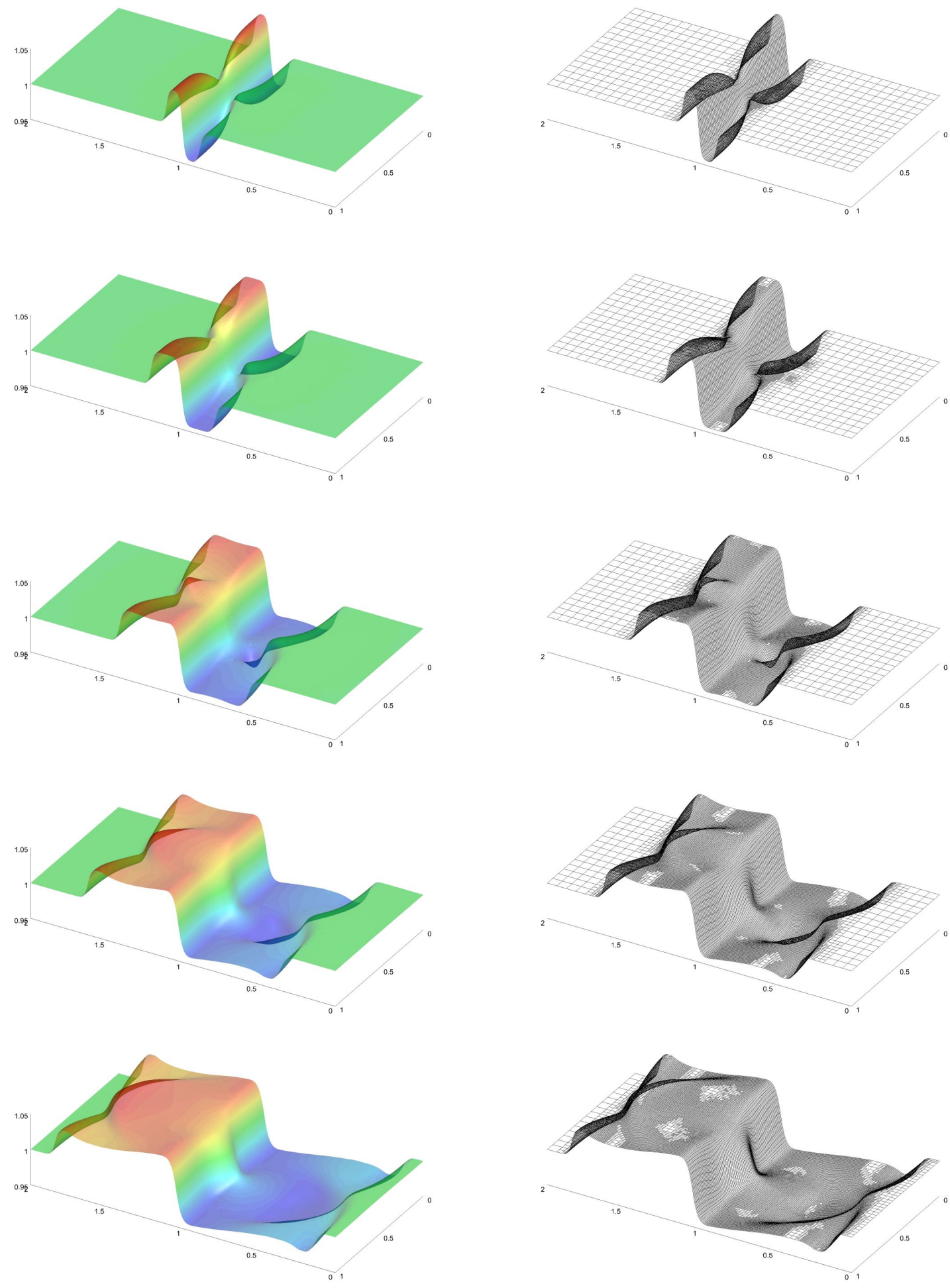

Figure 4: Example 2: Computed water surface $w(x, y, t)$ (left column) and corresponding quadtree grids (right column) for $t=0.1,0.2,0.4,0.6$, and 0.8 (from top to bottom) obtained using the non-well-balanced scheme. 

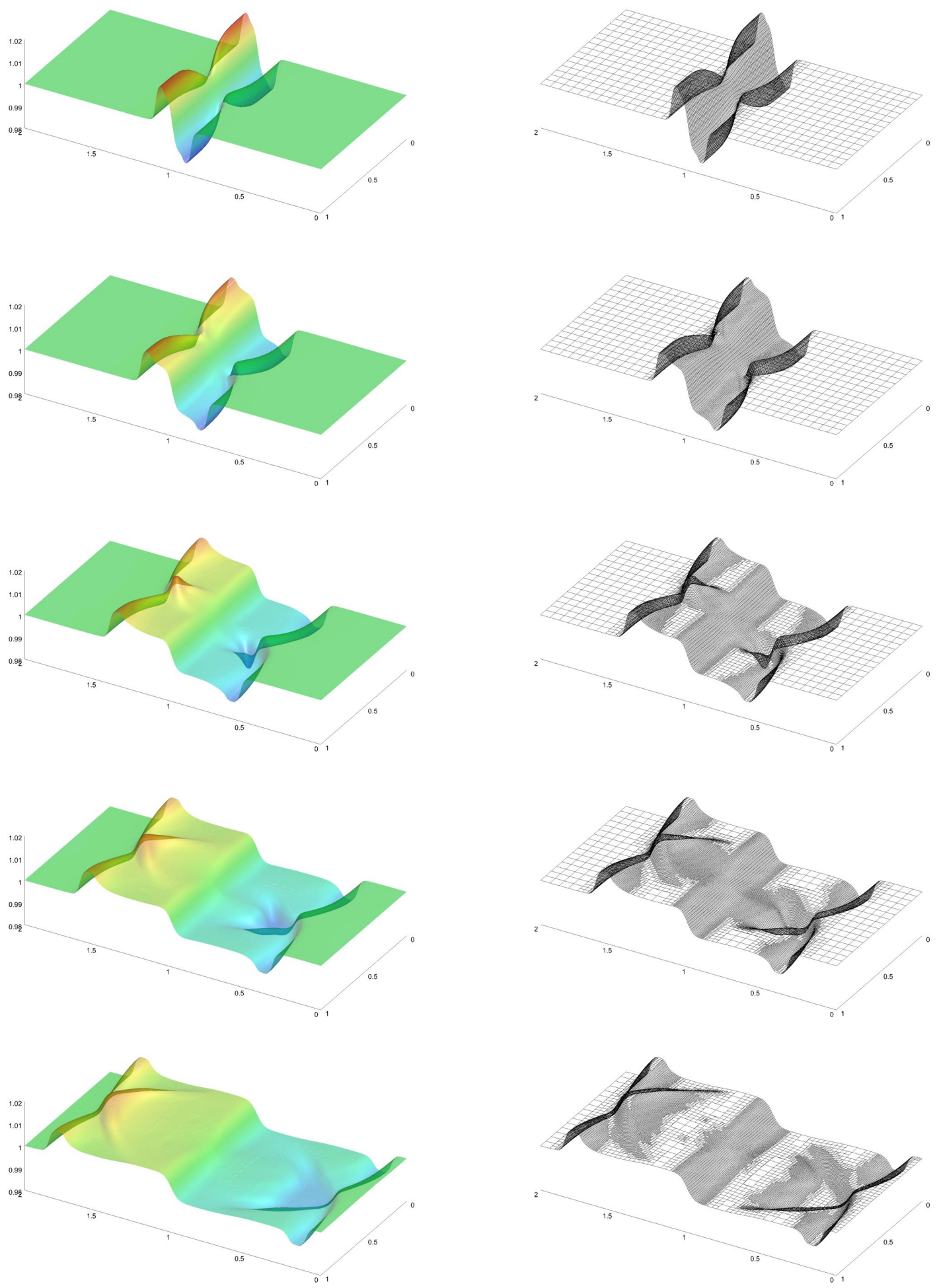

Figure 5: Example 2: Computed water surface $w(x, y, t)$ (left column) and corresponding quadtree grids (right column) for $t=0.1,0.2,0.4,0.6$, and 0.8 (from top to bottom) obtained using the well-balanced scheme. 


\section{Example 3 - Small perturbations of a stationary steady-state solution}

This numerical example tests the capability of the proposed scheme to capture small perturbations of a steady state solution [10, 11, 18, 27, 35, 38]. We choose a computational domain $[-2,2] \times[0,1]$ to prevent complicated boundary conditions. The initial conditions are

$$
\begin{aligned}
& w(x, y, 0)=\left\{\begin{array}{lc}
1.01, & 0.05<x<0.15, \\
1, & \text { otherwise },
\end{array} \quad u(x, y, 0)=v(x, y, 0) \equiv 0,\right. \\
& \rho(x, y, 0)= \begin{cases}1007, & 0.05<x<0.15, \\
\rho_{\circ}, & \text { otherwise },\end{cases}
\end{aligned}
$$

and the given bottom topography function in Example 2 .

We set boundary conditions similar to Example 2 for this test. $m=9$ is taken, and we set $C_{w \text {,seed }}=10^{-2}$ and $C_{\rho \text {, seed }}=10$ in (3.29) and (3.30). The non-well-balanced solution is computed until the final time $t=1.8$ and plot the snapshots of $w$ (left) and the quadtree grids (right) at times $t=0.6,0.9,1.2,1.5$, and 1.8 in the domain of $[0,2] \times[0,1]$ in Figure 6. The quadtree grid starts with 2,530 cells and reaches a maximum number of 13,804 cells during the time evolution. The well-balanced solution is illustrated in Figure 7, respectively. In this solution, the number of cells reaches the maximum of 10,222. Figure 7 demonstrates that the proposed well-balanced central-upwind quadtree scheme accurately captures small perturbations of the "lake-at-rest" steady state and that the symmetry of the solution is preserved.

\subsection{Example 4 - Sudden contraction with variable density inflow}

The last example is a modification of the example in [18, 23]. The purpose of this example is to demonstrate the positivity-preserving property of the proposed scheme.

We consider an open channel with a sudden contraction. The geometry of the channel is established on its contraction, where

$$
y_{b}(x)=\left\{\begin{array}{l}
0.5, \quad x \leq 1, \\
0.4, \quad \text { otherwise }
\end{array}\right.
$$

The computational domain is $[0,3] \times\left[0.5-y_{b}(x), 0.5+y_{b}(x)\right]$. Solid wall boundary conditions are imposed at all of the boundaries except for part of the left inflow boundary, with $u\left(0, y_{i}, t\right) \equiv 2$ and $\rho\left(0, y_{i}, t\right) \equiv 1007$, where $y_{i} \in[0.4,0.6]$. In addition, we set the right boundary to a zero-order extrapolation. The following initial conditions are prescribed:

$$
w(x, y, 0) \equiv 1, \quad u(x, y, 0)=v(x, y, 0) \equiv 0, \quad \rho(x, y, 0) \equiv \rho_{\circ} .
$$

In this example, we take $m=8$ refinement levels of the quadtree grid and set $C_{w}$, seed $=2$ and $C_{\rho \text {, seed }}=20$ in (3.29) and (3.30). We compute the solution with the bottom topography given in Example 2, where the water depth at the top of the humps is quite shallow, which makes it a good example to test the positivity-preserving property.

We compute the solution until the final time $t=1.9$ and plot the evolution of $w$ and $\rho$ at times $t=0.4,0.8,1.2,1.6$, and 1.9 in Figure 8 . The quadtree grid in this solution starts with a minimum of 298 and reaches a maximum of 9,928 cells. As one can see, the proposed centralupwind quadtree scheme preserves the positivity of the computed water depth and density. 

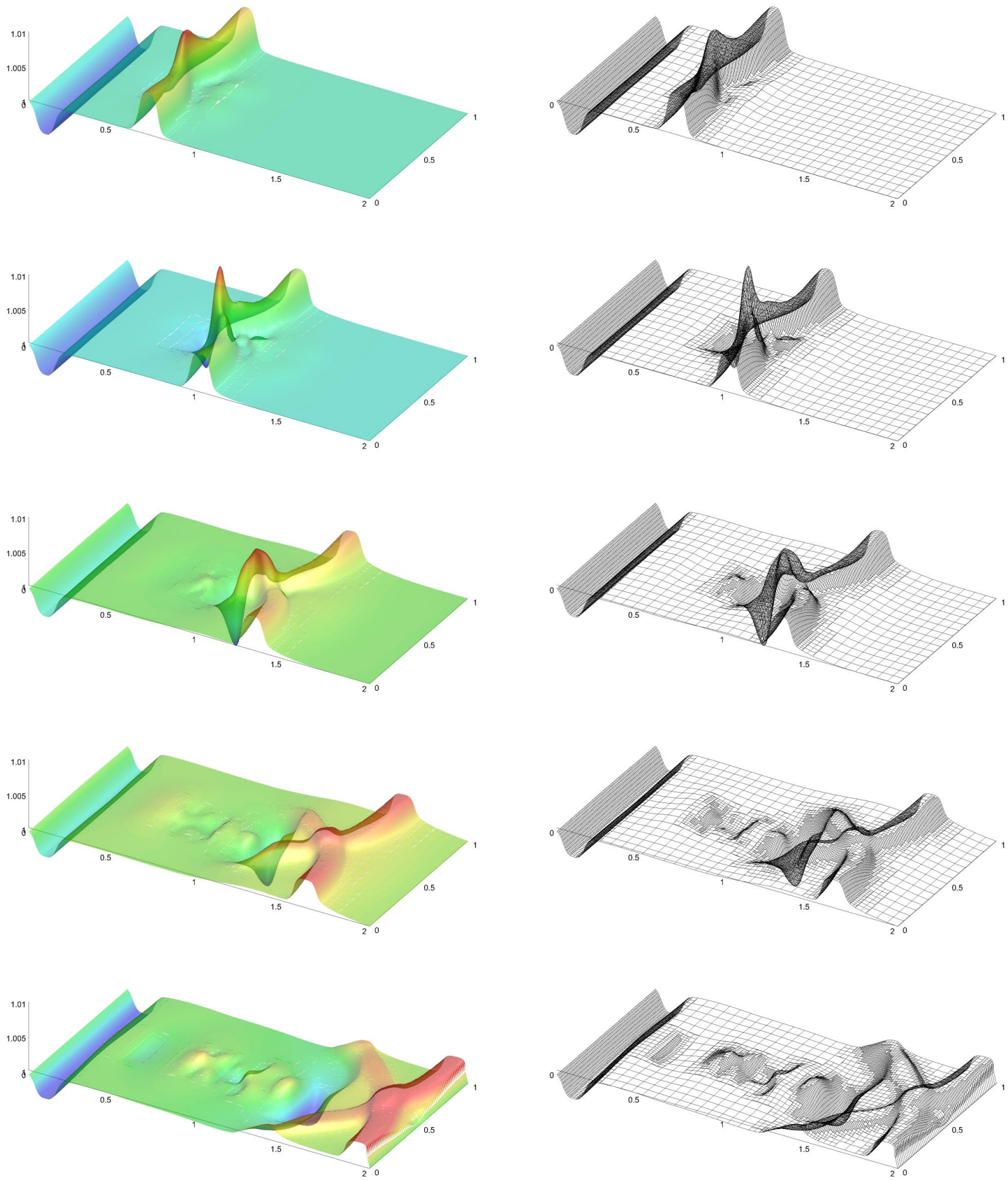

Figure 6: Example 3: Computed water surface $w(x, y, t)$ (left column) and corresponding quadtree grids (right column) for $t=0.6,0.9,1.2,1.5$, and 1.8 (from top to bottom) obtained using the non-well-balanced scheme. 

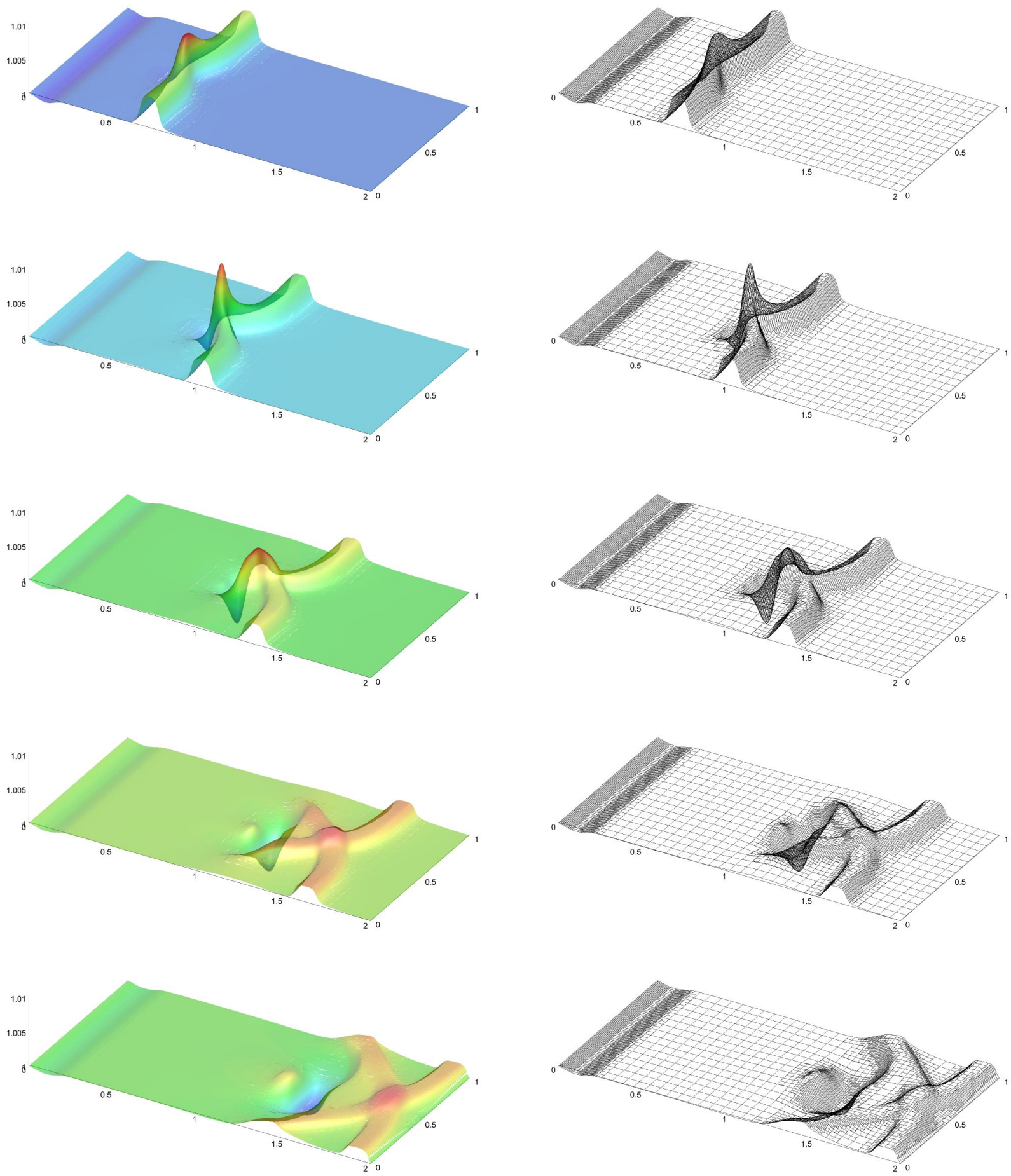

Figure 7: Example 3: Computed water surface $w(x, y, t)$ (left column) and corresponding quadtree grids (right column) for $t=0.6,0.9,1.2,1.5$, and 1.8 (from top to bottom) obtained using the well-balanced scheme.

\section{Conclusion}

An adaptive, well-balanced, positivity-preserving central-upwind scheme over quadtree grids for variable density shallow water equations has been presented. Four numerical examples have 

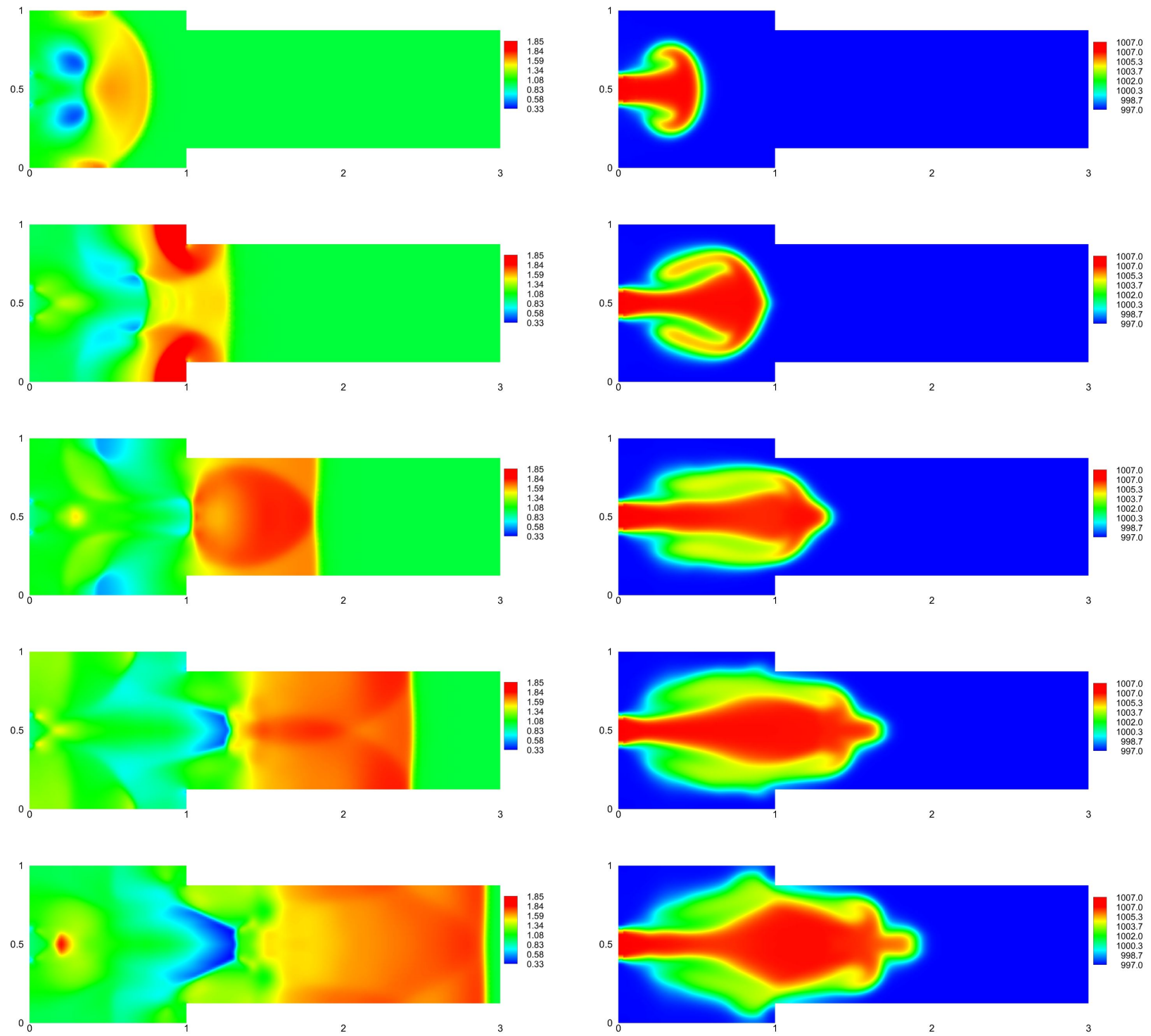

Figure 8: Example 4: Evolution of water surface $w(x, y, t)$ (left column) and density (right column) for $t=0.4,0.8,1.2,1.6$, and 1.9 (from top to bottom) obtained using the well-balanced scheme.

been used in order to verify the robustness and accuracy of the proposed scheme. These tests show symmetry preserving, well-balanced property, positivity-preserving, as well as adaptability of the coupled system. The results show that the proposed central-upwind quadtree scheme can improve the performance and efficiency of calculations compared to regular Cartesian grids.

\section{Acknowledgments}

The work of the authors was supported by NSERC grant 210717. The authors warmly thank Carlos Pars from the University of Mlaga for providing resources on the well-balanced property of the shallow water equations. The authors also thank Yangyang Cao and Philippe LeFloch from the Laboratoire Jacques-Louis Lions of Sorbonne Université for their useful discussions on 
the well-balanced property on conservation laws.

\section{References}

[1] K. Aizawa, K. Motomura, S. Kimura, R. Kadowaki, J. Fan, Constant time neighbor finding in quadtrees: An experimental result, in: 3rd International Symposium on Communications, Control and Signal Processing, 2008. ISCCSP 2008., IEEE, 505-510, 2008.

[2] H. An, S. Yu, Well-balanced shallow water flow simulation on quadtree cut cell grids, Adv. Water Resour. 39 (2012) 60-70.

[3] E. Audusse, F. Bouchut, M.-O. Bristeau, R. Klein, B. Perthame, A fast and stable wellbalanced scheme with hydrostatic reconstruction for shallow water flows, SIAM J. Sci. Comput. 25 (2004) 2050-2065.

[4] E. Audusse, M.-O. Bristeau, A well-balanced positivity preserving "second-order" scheme for shallow water flows on unstructured meshes, J. Comput. Phys. 206 (1) (2005) 311-333.

[5] A. Beljadid, A. Mohammadian, A. Kurganov, Well-balanced positivity preserving cellvertex central-upwind scheme for shallow water flows, Comput. \& Fluids 136 (2016) 193206.

[6] C. Berthon, F. Marche, A positive preserving high order VFRoe scheme for shallow water equations: a class of relaxation schemes, SIAM J. Sci. Comput. 30 (5) (2008) 2587-2612.

[7] A. Bollermann, G. Chen, A. Kurganov, S. Noelle, A well-balanced reconstruction of wet/dry fronts for the shallow water equations, J. Sci. Comput. 56 (2) (2013) 267-290.

[8] A. Bollermann, S. Noelle, M. Lukáčová-Medviďová, Finite volume evolution Galerkin methods for the shallow water equations with dry beds, Commun. Comput. Phys. 10 (2) (2011) $371-404$.

[9] A. G. L. Borthwick, R. D. Marchant, G. J. M. Copeland, Adaptive hierarchical grid model of water-borne pollutant dispersion, Adv. Water Resour. 23 (8) (2000) 849-865.

[10] S. Bryson, Y. Epshteyn, A. Kurganov, G. Petrova, Well-balanced positivity preserving central-upwind scheme on triangular grids for the Saint-Venant system, M2AN Math. Model. Numer. Anal. 45 (3) (2011) 423-446.

[11] S. Bryson, D. Levy, Balanced central schemes for the shallow water equations on unstructured grids, SIAM J. Sci. Comput. 27 (2005) 532-552.

[12] J. Burguete, P. García-Navarro, J. Murillo, Preserving bounded and conservative solutions of transport in one-dimensional shallow-water flow with upwind numerical schemes: Application to fertigation and solute transport in rivers, International Journal for Numerical Methods in Fluids 56 (9) (2008) 1731-1764.

[13] A. Chertock, M. Dudzinski, A. Kurganov, M. Lukáčová-Medvid'ová, Well-balanced schemes for the shallow water equations with Coriolis forces, Numerische Mathematik 138 (4) (2018) 939-973, ISSN 0029-599X. 
[14] A. Chertock, A. Kurganov, A second-order positivity preserving central-upwind scheme for chemotaxis and haptotaxis models, Numerische Mathematik 111 (2) (2008) 169-205, ISSN 0029-599X.

[15] A. Chertock, A. Kurganov, Y. Liu, Central-upwind schemes for the system of shallow water equations with horizontal temperature gradients, Numerische Mathematik 127 (4) (2014) 595-639, ISSN 0029-599X.

[16] Eleuterio F Toro, Shock-capturing methods for free-surface shallow fluids, Wiley and Sons Ltd, 2001.

[17] J. M. Gallardo, C. Parés, M. Castro, On a well-balanced high-order finite volume scheme for shallow water equations with topography and dry areas, J. Comput. Phys. 227 (1) (2007) $574-601$.

[18] M. A. Ghazizadeh, A. Mohammadian, A. Kurganov, An adaptive well-balanced positivity preserving central-upwind scheme on quadtree grids for shallow water equations, Computers $\&$ Fluids .

[19] S. Gottlieb, D. Ketcheson, C.-W. Shu, Strong stability preserving Runge-Kutta and multistep time discretizations, World Scientific Publishing Co. Pte. Ltd., Hackensack, NJ, 2011.

[20] S. Gottlieb, C.-W. Shu, E. Tadmor, Strong stability-preserving high-order time discretization methods, SIAM Rev. 43 (1) (2001) 89-112.

[21] D. M. Greaves, A. G. L. Borthwick, On the use of adaptive hierarchical meshes for numerical simulation of separated flows, Internat. J. Numer. Methods Fluids 26 (3) (1998) 303-322.

[22] J. Guo, A. G. L. Borthwick, Fluids Lattice Boltzmann method for variable density shallow water equations, Computers and Fluids 49 (1) (2011) 146-149, ISSN 0045-7930.

[23] M. E. Hubbard, On the accuracy of one-dimensional models of steady converging/diverging open channel flows, Internat. J. Numer. Methods Fluids 35 (2001) 785-808.

[24] L. Jiang, A. G. Borthwick, T. Krámer, J. Józsa, Variable density bore interaction with block obstacles, International Journal of Computational Fluid Dynamics 25 (4) (2011) 223-237.

[25] S. Khorshid, A. Mohammadian, I. Nistor, Extension of a well-balanced central upwind scheme for variable density shallow water flow equations on triangular grids, Computers and Fluids 156 (2017) 441-448, ISSN 0045-7930.

[26] A. Kurganov, Finite-volume schemes for shallow-water equations, Acta Numer. 27 (2018) 289-351.

[27] A. Kurganov, D. Levy, Central-upwind schemes for the Saint-Venant system, M2AN Math. Model. Numer. Anal. 36 (3) (2002) 397-425.

[28] A. Kurganov, C.-T. Lin, On the reduction of numerical dissipation in central-upwind schemes, Commun. Comput. Phys. 2 (1) (2007) 141-163. 
[29] A. Kurganov, S. Noelle, G. Petrova, Semidiscrete central-upwind schemes for hyperbolic conservation laws and Hamilton-Jacobi equations, SIAM J. Sci. Comput. 23 (3) (2001) $707-740$.

[30] A. Kurganov, G. Petrova, A second-order well-balanced positivity preserving centralupwind scheme for the Saint-Venant system, Commun. Math. Sci. 5 (1) (2007) 133-160.

[31] A. Kurganov, M. Prugger, T. Wu, Second-order fully discrete central-upwind scheme for two-dimensional hyperbolic systems of conservation laws, SIAM J. Sci. Comput. 39 (3) (2017) A947-A965.

[32] A. Kurganov, E. Tadmor, New high-resolution central schemes for nonlinear conservation laws and convection-diffusion equations, J. Comput. Phys. 160 (1) (2000) 241-282.

[33] W.-K. Lee, A. G. L. Borthwick, P. H. Taylor, A fast adaptive quadtree scheme for a twolayer shallow water model, J. Comput. Phys. 230 (12) (2011) 4848-4870.

[34] F. Z. Leighton, A. G. L. Borthwick, P. H. Taylor, 1-D numerical modelling of shallow flows with variable horizontal density, International Journal for Numerical Methods in Fluids (April 2009) (2009) n/a-n/a, ISSN 02712091.

[35] R. J. LeVeque, Balancing source terms and flux gradients in high-resolution Godunov methods: the quasi-steady wave-propagation algorithm, J. Comput. Phys. 146 (1) (1998) 346365 .

[36] Q. Liang, A. G. Borthwick, Adaptive quadtree simulation of shallow flows with wetdry fronts over complex topography, Computers \& Fluids 38 (2) (2009) 221-234, ISSN 00457930.

[37] X. Liu, J. Albright, Y. Epshteyn, A. Kurganov, Well-balanced positivity preserving centralupwind scheme with a novel wet/dry reconstruction on triangular grids for the Saint-Venant system, J. Comput. Phys. 374 (2018) 213-236.

[38] X. Liu, A. Mohammadian, A. Kurganov, J. A. Infante Sedano, Well-balanced centralupwind scheme for a fully coupled shallow water system modeling flows over erodible bed, J. Comput. Phys. 300 (2015) 202-218.

[39] F. Pascal, J. L. Marechal, Fast adaptive quadtree mesh generation, in: 7th International Meshing Roundtable, Citeseer, 211-224, 1998.

[40] S. Popinet, Gerris: a tree-based adaptive solver for the incompressible Euler equations in complex geometries, J. Comput. Phys. 190 (2) (2003) 572-600.

[41] S. Popinet, R. M. Gorman, G. J. Rickard, H. L. Tolman, A quadtree-adaptive spectral wave model, Ocean Model. 34 (1-2) (2010) 36-49.

[42] M. Ricchiuto, An explicit residual based approach for shallow water flows, J. Comput. Phys. 280 (2015) 306-344.

[43] B. Rogers, M. Fujihara, A. G. L. Borthwick, Adaptive Q-tree Godunov-type scheme for shallow water equations, Internat. J. Numer. Methods Fluids 35 (3) (2001) 247-280. 
[44] H. Samet, Algorithms for the conversion of quadtrees to rasters, Comput. Vision Graph. 26 (1) (1984) 1-16.

[45] H. Samet, Foundations of Multidimensional and Metric Data Structures, Morgan Kaufmann, first edn., 2006.

[46] H. Shirkhani, A. Mohammadian, O. Seidou, A. Kurganov, A well-balanced positivitypreserving central-upwind scheme for shallow water equations on unstructured quadrilateral grids, Comput. \& Fluids 126 (2016) 25-40. 